\title{
Evaluación del espacio fiscal para salud en Bolivia
}

\author{
Mauricio Matus-López, ${ }^{1}$ Desiderio Cansino Pozo, ${ }^{2}$ Camilo Cid Pedraza, ${ }^{3}$ Werner \\ Valdés Romero ${ }^{4}$
}

Forma de citar

Matus-López M, Cansino Pozo D, Cid Pedraza C, Valdés Romero W. Evaluación del espacio fiscal para salud en Bolivia. Rev Panam Salud Publica. 2018;42:e4. https:// doi.org/10.26633/RPSP.2018.4

RESUMEN Objetivo. Evaluar desde los puntos de vista técnico y político la capacidad de Bolivia para generar espacio fiscal para salud que le permita sostener los avances y cumplir el compromiso de un gasto público en salud de $6 \%$ del producto interno bruto (PIB).

Métodos. Se realizó una revisión de la evidencia internacional sobre espacio fiscal y sus mediciones. El análisis técnico se desarrolló a través del uso de estadísticas e informes oficiales de múltiples fuentes secundarias nacionales e internacionales. El análisis político y social se llevó a cabo mediante 20 entrevistas a representantes de organismos e instituciones.

Resultados. Existe capacidad para crear espacio fiscal en salud para Bolivia. Las fuentes con mayor factibilidad técnica y política son tres: el crecimiento económico vinculado al compromiso de repriorización del gasto público en salud, mejoras de las fuentes internas de recaudación a través de la reducción de la informalidad y reducción de los gastos tributarios. Con menos factibilidad se sitúa el financiamiento externo y el aumento de las contribuciones a la Seguridad Social.

Conclusiones. Bolivia está en condiciones de alcanzar un gasto público en salud de $6 \%$ del PIB. El tiempo en que logre esta meta depende de mantener la priorización del gasto en el sector, con el consenso de la mayoría social y política y en un marco de crecimiento y estabilidad económica.

Palabras clave Financiación de la atención de la salud; política financiera; cobertura universal; recursos en salud; Bolivia.

En 2016, Bolivia contaba con 10,9 millones de habitantes. Entre 2010 y 2014, su producto interno bruto (PIB) creció 5,3\% anual promedio, para alcanzar los 3 124,1 dólares estadounidenses per cápita. Este crecimiento, junto a las políticas sociales, le ha permitido mejorar casi todos sus indicadores. La tasa de pobreza disminuyó de $59,9 \%$ a 39,3\% de la población entre 2006 y

\footnotetext{
Departamento de economía, métodos cuantitativos e historia económica, Universidad Pablo de Olavide, Sevilla, España. Enviar la correspondencia a Mauricio Matus-López, mmatlop@ upo.es
}

2014, la pobreza extrema lo hizo de 37,7\% a $17,3 \%$, y la esperanza de vida aumentó más de diez años en solo dos décadas (1).

Pese a estos avances, el sector salud aún tiene problemas pendientes. Pese a que es uno de los países con mayor gasto público en salud (GPS), que corresponde a $4,6 \%$ del PIB en 2014, su gasto per cápita solo es de 142,27 dólares estadounidenses. Así,

\footnotetext{
2 Doctorando, Universidad Pablo de Olavide, Sevilla, España.

3 Organización Panamericana de la Salud, Washington D.C., Estados Unidos de América.

4 Consultor externo, La Paz, Bolivia.
}

todavía dos de cada tres bolivianos no tienen seguro de salud $(61,2 \%)$. Por otra parte, los que lo tienen, se distribuyen en un sistema fragmentado de más de una veintena de seguros públicos y privados (1-3).

En el año 2014, Bolivia, como el resto de los países miembros de la Organización Panamericana de la Salud, acordó elevar el GPS hasta 6\% del PIB (4), un valor que reduce de manera considerable la incidencia de la catástrofe financiera en los hogares (5) y permite avanzar hacia la salud universal. Para esto, es necesario evaluar si el país dispone de

Este es un artículo de acceso abierto distribuido bajo los términos de la licencia Creative Commons Attribution-NonCommercial-NoDerivs 3.0 IGO, que permite su uso, distribución y reproducción en cualquier medio, siempre que el trabajo original se cite de la manera adecuada. No se permiten modificaciones a los artículos ni su uso comercial. Al reproducir un artículo no debe haber ningún indicio de que la OPS o el artículo avalan a una organización o un producto específico. El uso del logo de la OPS no está permitido. Esta leyenda debe conservarse, junto con la URL original del artículo. 
capacidad de crear un espacio fiscal que lo financie.

El espacio fiscal (EF) se define como el conjunto de recursos adicionales que se pueden incorporar al presupuesto público para alcanzar una meta de desarrollo del país, sin comprometer su estabilidad o solvencia económico-financiera, y cuyas fuentes principales pueden ser de cuatro tipos: crecimiento económico, repriorización del gasto presupuestario, recaudación impositiva y eficiencia en el gasto público (6-10).

Se han realizado estudios de EF específicos para salud para una treintena de países de cuyos resultados se obtienen tres conclusiones: a) cada caso es diferente, no existen reglas generales y las especificidades de cada país condicionan los resultados, b) las fuentes de EF han de estar diversificadas y c) se aconseja que las fuentes de EF sean endógenas (7, 10-13).

El objetivo del presente estudio es evaluar las fuentes de creación de EF de Bolivia y estimar el margen existente para alcanzar esta meta de gasto.

\section{MATERIALES Y MÉTODOS}

Se utilizó una metodología cuantitativa y cualitativa.

Se identificaron las fuentes de espacio fiscal de acuerdo a una revisión de la bibliografía científica en las bases de datos PubMed, Web of Science, Scopus y Scielo. A esto se agregaron informes y documentos de trabajo de la Organización Mundial de la Salud (OMS) (14), Banco Mundial (BM) (15) y Organización Internacional del Trabajo (OIT) (16).

El análisis técnico se realizó utilizando información secundaria de fuentes nacionales e internacionales. Entre las primeras destacan el Instituto Nacional de Estadísticas (INE) (1), el Banco Central de Bolivia (BCB) (17), el Ministerio de Economía y Finanzas (MEF) (18-20), el Ministerio de Salud (MS) (21) y el Instituto Nacional de Seguros de Salud (INASES) (3). En las internacionales, se utilizaron las bases de datos de la OMS (2), BM (22), Fondo Monetario Internacional (FMI) (23) y la Organización para la Cooperación y Desarrollo Económico (OECD) (24).

El análisis sociopolítico se realizó a través de 20 entrevistas Anexo y encuestas a representantes institucionales, políticos y sociales. Esto incluyó al MS, MEF, Ministerio de Planificación del Desarrollo, Unidad de Análisis de Políticas Sociales y Económicas, Caja Nacional de Salud, bancada en la Asamblea Legislativa de Unidad Nacional (UN) y del Movimiento al Socialismo (MAS), Colegio Médico, Confederación Sindical Única de Trabajadores, fundaciones y expertos nacionales, entre otros. La muestra fue diseñada por un experto local, que realizó el trabajo de campo entre junio y julio de 2016 . Ochenta por ciento $(n=16)$ correspondieron a entrevistas presenciales, con una duración aproximada de 40 minutos y un total de 20 preguntas abiertas y cerradas. En el resto, 20\% $(n=4)$, se remitió un cuestionario con las mismas preguntas. Este fue el caso de las bancadas de UN y MAS. En las preguntas cerradas se utilizó una escala Lickert de grado de acuerdo/desacuerdo con distintas medidas y fuentes de financiamiento, tomando valor 1 en total desacuerdo y valor 10 en total acuerdo.

\section{RESULTADOS}

\section{Condiciones macroeconómicas de crecimiento estable}

Según las proyecciones de crecimiento del FMI 2015-2020, se estimó la evolución del GPS para tres escenarios: optimista, neutro y pesimista. Con base en la literatura internacional, se realizó la proyección a través de elasticidades del GPS con respecto al PIB $(11,13)$. En el escenario optimista, se consideró que el esfuerzo realizado en los últimos años se mantendrá en el tiempo. Por ello, se utilizó el promedio de 2004-2014. En el escenario pesimista se eliminaron de esta serie los dos valores más altos del mismo período. Por último, en el escenario pesimista, se utilizó la serie completa 1995-2014, con exclusión de los valores outlayer, lo que supone incorporar bajos valores de la década pasada. Las elasticidades fueron 1,63 para el escenario optimista, 1,25 para el neutro y 1,16 para el pesimista; esto resultó en incrementos de hasta 0,7 puntos del PIB, hasta 0,3 puntos del PIB y hasta 0,2 puntos del PIB, respectivamente (cuadro 1 ).

Desde la valoración sociopolítica, esta fuente tuvo una aceptación promedio de siete sobre diez puntos entre los entrevistados.

\section{Repriorización del gasto en salud}

En los países de ingresos mediobajos. La repriorización del GPS está condicionada al riesgo de generar nuevos problemas de financiamiento en otras necesidades importantes como educación o ayudas a la pobreza (6).

Los datos muestran que el GPS como porcentaje del GP total representa 9,7\% para el año 2013, muy por encima del porcentaje de $6,2 \%$ que registran los países de ingresos medio-bajos (2). Sin embargo, 9,7\% es aún un porcentaje bajo a nivel de la región de América Latina y el Caribe, si se tiene en cuenta que casi la mitad de los países de la región presentan porcentajes superiores a $15 \%$.

La creación de EF mediante la repriorización de presupuestos no se puede realizar por la vía de absolutos, sino por la vía de los incrementales (11), con énfasis en la distribución de los nuevos ingresos más que en la reordenación de los históricos.

El supuesto contemplado en los escenarios analizados en esta investigación implica que la distribución del presupuesto incremental se distribuya de forma similar al latinoamericano. Es decir, que el gasto en salud sea el resultado de aplicar 9,7\% al GP total del año anterior, más $15,1 \%$ sobre el incremento del gasto total para el año actual.

En un primer escenario, poco probable, se aplicaría ese $15,1 \%$ de inmediato. Un segundo escenario, más realista pero también optimista es que este criterio se aplique de manera progresiva. Como resultado, una repriorización del gasto puede generar un EF de 0,53 y 0,3 puntos del PIB, respectivamente. Esto confirma la evidencia internacional que señala que esta fuente de creación de EF es limitada por su estructura poco flexible y porque, aún sobre los incrementales, la decisión de disminuir el peso de un sector en beneficio de otro es difícil desde el punto de vista político.

\section{Nuevas fuentes de recaudación}

Cambios en impuestos generales. Los datos nacionales muestran que la recaudación tributaria no ha parado de crecer en la última década $(18,19)$. Para 2013, los ingresos por impuestos ascendieron a 27,6\% del PIB, 6,3 puntos más que la región $(21,3 \%)$ y a una distancia similar a la de los países de la OECD (34,1\%) (24).

Los ingresos tributarios presentan una composición que descansa primordialmente en los impuestos a las utilidades de las empresas (IUE) con 27,4\% del total, seguido del impuesto al valor 
CUADRO 1. Espacio fiscal para salud (proyección basada en condiciones macroeconómicas), Bolivia, 2012-2021

\begin{tabular}{|c|c|c|c|c|c|c|c|c|c|c|}
\hline & 2012 & 2013 & 2014 & 2015 & 2016 & 2017 & 2018 & 2019 & 2020 & 2021 \\
\hline Variación real del PIBa & 5,12 & 6,8 & 5,46 & 4,80 & 5,0 & 4,0 & 4,25 & 3,50 & 3,50 & 3,50 \\
\hline PIB (millones) ${ }^{b}$ & 193191 & 206321 & 217587 & 228031 & 239432 & 249010 & 259592 & 268679 & 278082 & 287815 \\
\hline Población (millones) & 10,24 & 10,4 & 10,57 & 10,74 & 10,9 & 11,07 & 11,24 & 11,4 & 11,56 & 11,73 \\
\hline \multicolumn{11}{|l|}{ Escenario optimista } \\
\hline Gasto público en salud (millones)b & 7288 & 8745 & 9934 & 10530 & 11386 & 12128 & 12967 & 13706 & 14487 & 15312 \\
\hline Gasto público en salud (\% del PIB) & $3,8 \%$ & $4,2 \%$ & $4,6 \%$ & $4,6 \%$ & $4,8 \%$ & $4,9 \%$ & $5,0 \%$ & $5,1 \%$ & $5,2 \%$ & $5,3 \%$ \\
\hline Gasto público en salud per cápitab & 712 & 841 & 940 & 980 & 1044 & 1096 & 1154 & 1202 & 1253 & 1305 \\
\hline \multicolumn{11}{|l|}{ Escenario neutro } \\
\hline Gasto público en salud (millones) ${ }^{\mathrm{b}}$ & 7288 & 8745 & 9934 & 10530 & 11188 & 11747 & 12371 & 12913 & 13477 & 14067 \\
\hline Gasto público en salud (\% del PIB) & $3,8 \%$ & $4,2 \%$ & $4,6 \%$ & $4,6 \%$ & $4,7 \%$ & $4,7 \%$ & $4,8 \%$ & $4,8 \%$ & $4,8 \%$ & $4,9 \%$ \\
\hline Gasto público en salud per cápita ${ }^{a}$ & 712 & 841 & 940 & 980 & 1026 & 1061 & 1101 & 1133 & 1165 & 1200 \\
\hline \multicolumn{11}{|l|}{ Escenario pesimista } \\
\hline Gasto público en salud (millones) ${ }^{\mathrm{b}}$ & 7288 & 8745 & 9934 & 10530 & 11140 & 11657 & 12231 & 12727 & 13243 & 13781 \\
\hline Gasto público en salud (\% del PIB) & $3,8 \%$ & $4,2 \%$ & $4,6 \%$ & $4,6 \%$ & $4,7 \%$ & $4,7 \%$ & $4,7 \%$ & $4,7 \%$ & $4,8 \%$ & $4,8 \%$ \\
\hline Gasto público en salud per cápitab & 712 & 841 & 940 & 980 & 1022 & 1053 & 1089 & 1116 & 1145 & 1175 \\
\hline
\end{tabular}

a Estimaciones de crecimiento: INE (1) y FMI (23).

b Bolivianos de 2016.

PIB, producto interno bruto.

Fuente: elaboración propia.

agregado (IVA) al mercado interno con $23,5 \%$, IVA al mercado externo con $22,3 \%$, y régimen complementario del IVA con $1 \%$, lo que en total significa el 46,8\% de los ingresos. Más atrás, los impuestos al consumo específico (ICE) conforman $6,1 \%(19)$.

Según la composición, la diferencia respecto a los países ricos se debe principalmente a impuestos directos. En la OCDE, la recaudación por estos alcanza 11,4\% del PIB, mientras que en América Latina y Caribe solo 5,5\%, y en Bolivia apenas 3,9\% (24).

La cuestión es muy importante, ya que la diferencia entre un tipo de impuesto y otro no es neutra para salud: los impuestos directos y progresivos son los que permiten favorecer la cobertura universal sin dañar los resultados en salud (25-27).

Sin obviar la enorme ventaja que posee el país de poder financiar parte de su gasto público a través de los recursos naturales, existe margen de recaudación de los impuestos directos a beneficios.

Una meta alcanzable puede ser la de reducir una cuarta parte la brecha impositiva sobre renta y beneficios con los países de la región mediante el aumento de esta última hasta 4,3\% del PIB. Otro escenario más optimista sería igualar de forma progresiva esta recaudación para que en 2021 disminuya la brecha a la mitad; es decir, que se eleve hasta $4,7 \%$ del PIB.
La creación de EF en cada escenario puede alcanzar un promedio entre $0,4 \mathrm{y}$ 0,8 puntos del PIB, respectivamente. Además, desde la valoración sociopolítica, tuvo una aceptación promedio de ocho sobre diez puntos.

Impuestos sobre bienes sanitarioespecíficos. Estos impuestos se refieren básicamente a tres tipos: sobre tabaco, sobre bebidas alcohólicas y sobre alimentos con alto contenido de azúcares o grasas. Se denominan sanitarioespecíficos (conocidos en la literatura en inglés como sin-taxes y earnmarked) porque tienen una doble finalidad: aumentar la recaudación tributaria que se puede destinar directamente a salud y, por otro lado, desincentivar el consumo nocivo y, por tanto, reducir el coste futuro en sanidad.

Respecto a las bebidas alcohólicas, en términos reales, las tasas del ICE se han mantenido constantes o han disminuido sobre la mayoría de productos, excepto para la cerveza. Dada la elasticidad que presentan (28) y los niveles actuales de impuestos, un alza impositiva no tendría impacto significativo en términos de EF, pero sí tendría efectos positivos en términos de la reducción del gasto en tratamientos de enfermedades relacionadas.

Respecto al tabaco, presenta una tasa impositiva de las más bajas de América Latina y lejos de los niveles de Francia,
España o Chile, por lo que existe margen para crear EF mediante esta fuente.

La recaudación de impuestos al tabaco ascendió a los 121,9 millones de bolivianos (Bs) en 2013 (14), lo que equivale a 8,9\% del total de ingresos por impuestos específicos internos, $0,2 \%$ de los ingresos tributarios y solo $0,05 \%$ del PIB. Si se asume la elasticidad señalada de $-0,85$ (29) y un incremento de $20 \%$ del precio, la recaudación crearía un EF del 2\% de los impuestos. $\mathrm{Si}$ bien es un porcentaje bajo en términos del PIB, se traducen en 2,4 millones de Bs extra para el presupuesto.

Cabe destacar, sin embargo, un posible efecto de incentivos al mercado negro en el comercio de estos bienes. No obstante, no se dispone de estimaciones de elasticidad-precio de este fenómeno en Bolivia.

En la valoración sociopolítica, el alza de impuestos al tabaco y bebidas alcohólicas nacionales alcanzó un nivel medio de apoyo de siete sobre diez puntos. En los impuestos a bebidas importadas, el acuerdo fue más alto; de nueve sobre diez puntos. Las opiniones favorables se basaron en los beneficios económicos y sociales de reducir el consumo de estos productos.

Impuestos sobre recursos naturales. En Bolivia, los impuestos sobre los recursos naturales suponen una de las principales fuentes de financiamiento del GP. Solo el impuesto directo a los 
hidrocarburos (IDH) representó $27 \%$ del total de los ingresos tributarios.

El IDH es una clara fuente de EF con margen para seguir generando recursos. Sin embargo, su flujo depende de las variaciones internacionales de los precios de las materias primas.

En materia de EF para salud, los recursos provenientes de los hidrocarburos pueden facilitar la meta de $6 \%$ del GPS. En 2014, solo el IDH alcanzó 6,8\% del PIB. Bastaría dirigir a salud un aumento de menos de 10\% sobre la recaudación de este impuesto para aumentar el GPS en más de medio punto del PIB y dejar al país muy cerca de la meta. Todo descansa en la factibilidad política y el impacto de una decisión de este tipo sobre las decisiones empresariales de producción, algo que escapa al presente análisis.

Aportes a la seguridad social. La recaudación de contribuciones sociales en el país es muy baja, representando apenas $1,4 \%$ de su PIB, mientras que la media en América Latina alcanza 3,8\% del PIB y 9\% del PIB en los países de la OCDE.

Existen dos explicaciones directas de esta baja recaudación. La primera es la decisión política de tener un sistema donde se carga todo el peso de las contribuciones a empleadores, sin aportaciones obligatorias de los trabajadores, como ocurre en la mayoría de los países con sistemas de seguridad social que financian salud.

La segunda explicación está motivada por el alto nivel de informalidad en el mercado laboral, en torno a $75 \%(30,16)$.

Un esfuerzo que implique reducir en diez puntos la informalidad podría elevar las afiliaciones entre $15,6 \%$ y $24,4 \%$, es decir, entre 0,22 y 0,34 puntos del PIB sin modificar las tasas de cotización.

La modificación de las tasas de cotización está casi excluida. Los entrevistados estuvieron muy en desacuerdo con esta medida: apenas logró un apoyo medio de dos sobre diez puntos posibles.

Así, los márgenes de EF por la vía de aportes a la seguridad social pueden ser amplios, si se reduce la informalidad y se favorece un esquema de aporte solidario (30), pero no parece factible aumentar las tasas de cotización.

\section{Aumento de la eficiencia en la recaudación y en el gasto}

En este apartado se analizan dos tipos de eficiencia, tal como se señala en el marco analítico de OPS (9): los gastos tributarios como eficiencia en la recaudación y eficiencia en la gestión del sistema de salud.

Eficiencia en la recaudación: gastos tributarios. El gasto tributario es el conjunto de excepciones sobre las normas tributarias generales con el fin de alcanzar determinados objetivos estratégicos para la economía. Según el MEFP, los gastos tributarios alcanzaron 1,3\% del PIB en 2013, lo que supone un aumento de $17,7 \%$ respecto al 2011 , pero una reducción respecto a $2005(18,32,33)$.

La generación de EF que pasa por la eliminación de parte de los gastos tributarios alcanzaría 1,3\% del PIB. Sin embargo, hay que considerar qué parte de este gasto tributario conviene mantener o eliminar.

El mismo MEFP plantea dos consideraciones (20). La primera son las exenciones de la alícuota adicional al IUE de las cooperativas mineras, que gravan beneficios extraordinarios y, aunque sean cooperativas, gravan también la explotación de un recurso no renovable. La segunda cuestión se refiere al escaso efecto de los incentivos destinados a la promoción económica de algunas regiones, cuyo resultado fue el incremento de la complejidad de la normativa tributaria.

Por último, la industria exportadora, causante de casi la mitad de los gastos tributarios, debe someterse a un análisis profundo, en especial a lo referido con aquella parte que de escaso valor agregado.

Siguiendo las directrices del MEFP, y mediante la reducción de $10 \%$ los gastos tributarios a la exportación, se podría generar un margen de entre $0,07 \%$ y $0,1 \%$ del PIB.

Desde la valoración sociopolítica, existe un alto nivel de acuerdo con la reducción de los gastos tributarios. En una escala de uno a diez, los entrevistados promediaron un nivel de ocho. No obstante, los poderes de negociación de los agentes son muy variados, y en este particular, resultan muy relevantes.

Eficiencia en la gestión: gasto en salud. La eficiencia en el GPS es una fuente para la casi completa totalidad de estudios sobre EF. En la práctica, la estimación de un indicador de eficiencia es compleja y, por último, su evaluación pasa por comparaciones entre países o análisis nacionales acotados a determinados servicios o regiones.

La evidencia señala que un mayor GPS mejora los indicadores de salud y es más efectivo cuando su recaudación se basa en impuestos directos o mecanismos de riesgo compartido $(25,34)$.

En base a lo anterior, un cálculo simple y directo es comparar GP y tasa de mortalidad infantil para una serie de países en el mundo. De acuerdo a esto, Bolivia registra tasas de mortalidad similares a países con niveles de ingresos similares, por lo que se puede interpretar que su desempeño en salud es normal dado los recursos públicos aplicados (2).

En un análisis por el modelo econométrico envolvente de datos aplicado a 165 países, Bolivia ocupa el lugar 28 entre los 45 de ingresos medio-bajos. Esto es algo por encima del promedio de su grupo, aunque por detrás de los países latinoamericanos del mismo conjunto (35).

Un tercer análisis considera una frontera de posibilidades de producción cóncava. Es decir, que las posibilidades de ganancias marginales de productividad son menores mientras mayor es el gasto (36). En este caso, Bolivia ocupó el lugar 62 de 191 países. Debido a la evidencia anterior, aunque logre mejorar su eficiencia en 5\%, el EF generado sería de aproximadamente $0,1 \%$ del PIB.

En otro sentido, la eficiencia se aumenta también en la optimización de los modelos de financiamiento y aseguramiento. La existencia en el país de una veintena de seguros de salud que conviven con un porcentaje importante de la población sin asegurar es un signo evidente de ineficiencia (3).

Por último, el desarrollo y fortalecimiento de un sistema de atención de salud organizado en redes integradas y basado fuertemente en el primer nivel, puede genera importantes ganancias de eficiencia. Esto puede ser apoyado con políticas financieras que promuevan la implementación de sistemas de pago adecuados.

La mejora de la eficiencia del gasto público cuenta con un apoyo promedio de nueve sobre diez puntos.

\section{Financiación externa}

Se analizan dos fuentes de EF: la deuda pública y las donaciones y cooperación internacionales.

Deuda pública. Bolivia es una economía que vio descender su deuda pública desde 63,5\% del PIB en 2003 hasta 14,5\% en 2011. Esto le otorga una adecuada posición para afrontar sus compromisos financieros y tener capacidad de 
CUADRO 2. Espacio fiscal para salud (fuente, factibilidad técnica, aceptación política/social y valoración), Bolivia, 2015-2020

\begin{tabular}{|c|c|c|c|}
\hline Fuente & Técnica & Factibilidad política y social & Sugerida \\
\hline $\begin{array}{l}\text { Condiciones macroeconómicas de } \\
\text { crecimiento estable }\end{array}$ & $\begin{array}{l}\text { Alta, con un margen promedio de tres escenarios (pesimista, neutro y } \\
\text { optimista) desde } 0,17 \text { a } 0,7 \text { puntos del PIB }\end{array}$ & Alta & $\begin{array}{l}\text { Continuar de forma } \\
\text { conjunta }\end{array}$ \\
\hline Repriorización & $\begin{array}{l}\text { Media, con un margen de } 0,3 \text { a } 0,53 \text { puntos del PIB con repriorización } \\
\text { extraordinaria }\end{array}$ & Media & \\
\hline \multicolumn{4}{|l|}{ Nuevos ingresos } \\
\hline Impuestos generales & $\begin{array}{l}\text { Alta en impuestos directos a beneficios y renta que puede alcanzar } \\
\text { un promedio de } 0,4 \text { a 0,8 de punto del PIB }\end{array}$ & $\begin{array}{l}\text { Muy Alta } \\
\text { en impuestos sobre rentas altas } \\
\text { Media } \\
\text { en impuestos a empresas }\end{array}$ & $\begin{array}{l}\text { Evaluar impuestos y } \\
\text { disminuir informalidad }\end{array}$ \\
\hline Impuestos sanitario- específicos & $\begin{array}{l}\text { Baja, en términos directos de recaudación de poco más de 0,04 } \\
\text { puntos. Puede ser alta en reducción de costos futuros }\end{array}$ & $\begin{array}{l}\text { Alta } \\
\text { en tabaco y en alcoholes } \\
\text { nacionales e importados }\end{array}$ & Implementar \\
\hline Impuesto a recursos naturales & $\begin{array}{l}\text { Alta, con un margen muy flexible que puede superar fácilmente } 0,5 \\
\text { puntos del PIB, pero muy fluctuante }\end{array}$ & Alta & Solución mixta \\
\hline $\begin{array}{l}\text { Contribuciones a la seguridad } \\
\text { social }\end{array}$ & $\begin{array}{l}\text { Media, con un margen entre } 0,2 \text { y } 0,3 \text { puntos del PIB con reducción } \\
\text { de la informalidad }\end{array}$ & $\begin{array}{l}\text { Baja } \\
\text { a trabajadores o empleadores }\end{array}$ & $\begin{array}{l}\text { Disminuir la } \\
\text { informalidad }\end{array}$ \\
\hline \multicolumn{4}{|l|}{ Aumentos de eficiencia } \\
\hline En tributación & Media, con un margen de 0,07 a 0,1 puntos del PIB & Alta & Evaluar \\
\hline En gasto en salud & $\begin{array}{l}\text { Media, con un margen inicial de } 0,1 \text { punto y con espacio en } \\
\text { unificación de seguros }\end{array}$ & Muy Alta & Seguro universal \\
\hline \multicolumn{4}{|l|}{ Financiamiento externo } \\
\hline Endeudamiento público & Alta, pero desaconsejable técnicamente & Baja & Descartada \\
\hline Donaciones internacionales & Baja y desaconsejable técnicamente & Media & Descartada \\
\hline Emisión monetaria & Baja y desaconsejable técnicamente & Ninguna & Descartada \\
\hline
\end{tabular}

PIB, producto interno bruto.

Fuente: elaboración propia.

endeudamiento. Pero ampliar el EF en salud mediante el endeudamiento se traduciría en adelantar en el tiempo los recursos que se recaudan desde el futuro al presente con un costo en intereses.

Desde la valoración sociopolítica, existe poca aceptación al endeudamiento como fuente de EFS, sobre todo si los recursos se quieren destinar a salarios. Sin embargo, el nivel de acuerdo mejora cuando estos recursos se conciben para ser invertidos en infraestructuras en el sector, como la construcción de nuevos hospitales.

Donaciones y cooperación internacional. Como en otros países, la incertidumbre de los flujos y su variabilidad hacen desaconsejable esta fuente de creación de EF $(7,11,13)$.

\section{DISCUSIÓN}

La revisión de la evidencia internacional muestra que, en la mayoría de los casos estudiados, existe capacidad de generar EF. Bolivia muestra resultados en esta misma línea (cuadro 2). El país cuenta con posibilidades de aumentar el GPS sin dañar la sostenibilidad económica.

En el aspecto técnico, los resultados coinciden con la evidencia internacional. El crecimiento económico es una de las fuentes que convoca mayor consenso político y social, y representa un mayor margen de recaudación. Si continúa su tendencia creciente a la vez que se mantiene la priorización del GP, esta fuente llevará a alcanzar la meta de $6 \%$ en GPS. La repriorización del GP puede reducir el tiempo de alcance esta meta.

Si bien la explotación de hidrocarburos supone una importante fuente de recursos para el Estado, no se pueden dejar de ignorar la generación de ingresos nuevos mediante los impuestos directos, sanitaros o la reducción de la informalidad laboral. Esta última, una de las fuentes más alejadas de la influencia del sector salud.

El sistema de aseguramiento presenta ineficiencias $y$, aunque el sistema público muestra avances en el acceso, tres de cada cinco personas no están cubiertas por un seguro y las economías de escala se pierden en un entramado de microsistemas que generan desigualdades en un ámbito sensible como es la salud.

Cabe destacar cuatro limitaciones del estudio. Primero, los cálculos no cuantifican el costo económico de la cobertura universal, sino las posibilidades de financiamiento del GPS. Segundo, el número y metodología de las entrevistas no valora el poder de los agentes, sino que entrega información sobre los grados de aceptación o rechazo de las fuentes de financiamiento. Tercero, no se realiza una valoración de los plazos en que se podría implementar el EF. Por último, al ser un estudio de financiamiento, no analiza la distribución del gasto por niveles o territorios ni su impacto distributivo.

\section{CONCLUSIONES}

Si bien el desafío de alcanzar un GPS de $6 \%$ del PIB requiere de un esfuerzo considerable, se plantea como un logro alcanzable. Las fuentes de financiamiento presentan margen por la vía del crecimiento económico, pero también de la mejora de la recaudación de 
impuestos y de la mayor eficiencia del sistema. Por último, las personas y los procesos organizacionales del sector, serán fundamentales para que estos recursos se traduzcan en un acceso más equitativo a la salud en Bolivia.

1. Instituto Nacional de Estadística de Bolivia. Información estadística. Disponible en: http:/ / www.ine.gob.bo Acceso el 1 de junio de 2016.

2. World Health Organization. Global Health Observatory Data Repository. Disponible en: http:/ / bit.ly/1Kv0EfJ Acceso el 20 de junio de 2016.

3. Instituto Nacional de Seguros de Salud de Bolivia. Anuario estadístico 2014. Disponible en: http://inases.gob.bo/ normas-2/anuario-estadistico/ Acceso el 10 de junio de 2016.

4. Organización Panamericana de la Salud. Resolución CD53. R14. Estrategia para el acceso universal a la salud y la cobertura universal de salud. 53o. Consejo Directivo, 66a. Sesión del Comité Regional de la OMS para las Américas. Washington, D.C., EUA, del 29 de septiembre al 3 de octubre del 2014. Disponible en: http:// bit.ly/1KzWv7n

5. Ke X, Saksena P, Jowett M, Indikadahena Ch, Kutzin J, Evans D. Exploring the thresholds of health expenditure for protection against financial risk. World Health Report, Background Paper, 2010;19. Disponible en: http://www.who.int/healthsystems/ topics / financing/healthreport/19THEthresv2.pdf?ua $=1$

6. Heller P. Back to Basics. Fiscal space: what it is and how to get it. Finance and Development, International Monetary Fund 2005;42(2). Disponible en: http://bit. ly/1LNX528

7. Tandon A, Cashin C. Assessing public expenditure on health from a fiscal space perspective. Washington, D.C.: World Bank; 2010. Disponible en: http://bit. ly/1Pt6AHl

8. Ostry JD, Ghosh AR, Kim JI, Qureshi MS. Fiscal space. IMF Staff Position Paper. 2010;SPN/10/11,1. Disponible en: http:/ / clar.in/1VaAwtw

9. Organización Panamericana de la Salud (OPS). Espacio fiscal para el aumento de la prioridad de salud en el gasto público en la Región de las Américas. Washington D.C: OPS/OMS; 2015. Disponible en: http://bit.ly/1Wzk5MM

10. Barroy H, Sparkes S, Dale E. Assessing fiscal space for health expansion in low and middle-income countries: A review of the evidence. Health Financing Working Paper. 2016;3. Disponible en: http://apps. who.int/iris/bitstream/10665/251904/1/ WHO-HIS-HGF-HFWorkingPaper-16.3eng.pdf?ua $=1$

11. Matus-López M, Prieto L, Cid C. Espacio fiscal para salud en Perú. Rev Panam Salud Pública. 2016;4(1):64-9.
Conflictos de interés. Ninguno declarado por los autores.

Financiamiento. El presente estudio fue financiado por la Organización Panamericana de la Salud.

\section{REFERENCIAS}

12. Durán-Valverde F, Pacheco JF. Fiscal space and the extension of social protection. Lessons learnt from developing countries. ESS Paper Series SECSOC. 2012;33. Disponible en: http: / / bit.ly/1iM3tAd

13. Sharma J. An assessment of fiscal space for health in Bhutan: Fiscal space for health in Bhutan. Int J Health Plann Manage. 2015. doi:10.1002/hpm.2295

14. World Health Organization (WHO). WHO report on the global tobacco epidemic. Raising taxes on tobacco. Luxemburg: WHO; 2016. Disponible en: http://bit. ly/1HdHZRY

15. World Bank. Purchasing power parities and the real size of world economies. A comprehensive report of the 2011 International Comparison Program. Washington D.C.: World Bank; 2016. doi: 10.1596/ 978-1-4648-0329-1

16. Organización Internacional del Trabajo (OIT). Panorama laboral temático. Transición a la formalidad en América Latina 2014. Lima: Oficina Regional para América Latina y el Caribe; 2014. Disponible en: http:/ / bit.ly/1ORKEI3

17. Banco Central de Bolivia (BCB). Boletines estadísticos 2004-2015. 2016. Disponible en:https://www.bcb.gob.bo/?q=pub_boletinestadistico

18. Ministerio de Economía y Finanzas Públicas de Bolivia. Tributación sobre Industria Extractiva en Bolivia. Conferencia "Tributación de las industrias extractivas en la Región Andina". Lima: Viceministerio de Política Tributaria; 2014. Disponible en: https://www.imf.org/external/spanish/ $\mathrm{np} /$ seminars/2014/natres/pdf/rios3.pdf

19. Ministerio de Economía y Finanzas Públicas de Bolivia. Boletín de ingresos y gastos tributarios. 2016:5. Disponible en: http:// medios.economiayfinanzas.gob.bo/VPT/ documentos/Boletines_Ingresos_Tributarios/BIyGT_Nro_5.pdf

20. Ministerio de Economía y Finanzas Públicas de Bolivia. Política Tributaria. 2016. Disponible en: http://www.economiayfinanzas.gob.bo/viceministerio-de-politicatributaria.html

21. Ministerio de Salud de Bolivia. Programas de salud. 2016. Disponible en: https:/ / www. minsalud.gob.bo/programas-de-salud

22. World Bank. World Development Indicators. 2016. [en línea]. Disponible en: http:/ / data.worldbank.org/data-cata$\log$ /world-development-indicators Acceso el 1 de junio de 2016.

23. Fondo Monetario Internacional. World Economic Outlook Database. 2016. [en línea] Disponible en: http://www.imf. org/external/pubs/ft/weo/2016/01/
Declaración. Las opiniones expresadas en este manuscrito son responsabilidad de los autores y no reflejan necesariamente los criterios ni la política de la RPSP/PAJPH y/o de la OPS. weodata/index.aspx Acceso el 1 de mayo de 2016.

24. Organisation for Economic Co-operation and Development (OECD), Economic Commission for Latin America and the Caribbean (ECLAC), Inter-American Center of Tax Administrators (CIAT), InterAmerican Development Bank (IDB). Revenue statistics in Latin America and the Caribbean. Paris: OECD Publishing; 2015. doi:10.1787/rev_lat-2015-en-fr.

25. Moreno-Serra R, Smith PC. Does progress towards universal health coverage improve population health? Lancet. 2012; 380(9845):917-23. doi: 10.1016/S0140-6736 (12)61039-3.

26. Reeves A, Gourtsoyannis Y, Basu S. Financing universal health coverage effects of alternative tax structures on public health systems: cross-national modelling in 89 low-income and middle-income countries. Lancet. 2015;386(9990):274-80. doi: 10.1016/S0140-6736(15)60574-8.

27. Yates R. Universal health coverage: Progressive taxes are key. Lancet. 2015; 386(9990):227-9. doi:10.1016/S0140-6736 (15)60868-6.

28. Wagenaar AC, Salois MJ, Komro KA. Effects of beverage alcohol price and tax levels on drinking: a meta-analysis of 1003 estimates from 112 studies. Addiction. 2009;104:179-90.

29. Alcaráz V. Economía del Control del Tabaco en los Países del Mercosur y Estados Asociados: Bolivia, Organización Panamericana de la Salud, 2006. Disponible en: http://iris.paho.org/xmlui/handle/1234 $56789 / 2810$

30. Vargas M, Garriga S. Explaining inequality and poverty reduction in Bolivia. IMF Working Paper. 2015;WP/15/265. Disponible en: https://www.imf.org/external/ pubs/ft/wp/2015/wp15265.pdf

31. Titelman $\mathrm{D}$, Cetrángolo $\mathrm{O}$, Acosta OL. Universal health coverage in Latin American countries: How to improve solidaritybased schemes. Lancet. 2015;385(9975): 1359-63. doi:10.1016/S0140-6736(14)61780-3

32. Villela L, Lemgruber A, Jorratt M. Gastos tributarios: la reforma pendiente. Trimestre Fiscal. 2010;92:79-119. Disponible en: http://bit.ly/1WiDGxM

33. Trigueros MP. Gastos tributarios en América Latina: 2008-2012. Centro Interamericano de Administraciones Tributarias CIAT. 2014. Disponible en: http:// www.ciat.org/index.php/es/productos$\mathrm{y}$-servicios/publicaciones/documentosde-trabajo / 3582-dt-02-2014-gastostributarios-en-america-latina-2008-2012. html 
34. Bokhari FAS, Gai Y, Gottret P. Government health expenditures and health outcomes. Health Econ. 2007;16(3):257-73. doi: 10.1002/hec.1157

35. González E, Cárcaba A, Ventura J. Value efficiency analysis of health systems: Does public financing play a role? J Public Health. 2010;18(4):337-50. doi:10.1007/ s10389-009-0311-4.

36. Kumbhakar SC. Efficiency and productivity of world health systems: where does your country stand? Appl Econ. 2010;
42(13):1641-59. doi:10.1080/000368407017 21588.

Manuscrito recibido el 24 de mayo de 2017. Aceptado para publicación, tras revisión, el 17 de julio de 2017.

ABSTRACT Objective. To assess from a technical and political point of view the capacity of Bolivia to generate fiscal space for health that allows it to sustain progress and fulfill the commitment of a public health expenditure of $6 \%$ of gross domestic product.

\section{Evaluation of fiscal space for health in Bolivia}

Keywords Healthcare financing; financial policy; universal coverage; health resources; Bolivia.
RESUMO

\section{Avaliação do espaço fiscal para a saúde na Bolívia}

Palabras-chave
Objetivo. Avaliar do ponto de vista técnico e político a capacidade da Bolívia para gerar espaço fiscal para a saúde que lhe permita sustentar o progresso e cumprir o compromisso de uma despesa de saúde pública de $6 \%$ do Produto Interno Bruto (PIB). Métodos. Foi feita uma revisão da evidência internacional sobre o espaço fiscal e suas medições. A análise técnica foi desenvolvida através do uso de estatísticas e relatórios oficiais de múltiplas fontes secundárias nacionais e internacionais. A análise política e social foi realizada através de 20 entrevistas com representantes de organizações e instituições.

Resultados. Existe capacidade para criar espaço fiscal em saúde para a Bolívia. As fontes com maior viabilidade técnica e política são três: crescimento econômico ligado ao compromisso de desajustar as despesas públicas em saúde, melhorias nas fontes internas de receita através da redução da informalidade e redução de despesas tributárias. Com menos viabilidade é o financiamento externo e o aumento das contribuições para a Segurança Social.

Conclusões. A Bolívia é capaz de alcançar gastos de saúde pública de $6 \%$ do PIB. O tempo que atinge esse objetivo depende da manutenção da priorização dos gastos no setor, com o consenso da maioria social e política, tudo em um quadro de crescimento econômico e estabilidade.

Financiamento da assistência à saúde; política financeira; cobertura universal; recursos en saúde; Bolívia. 